\title{
I Introduction
}

THE study of American economic history has been revolutionised in the past twenty years. Starting from the work of Kuznets [1952], far more data about the American economy in the nineteenth century have been collected than was believed possible only a few years ago. And starting essentially from the work of Conrad and Meyer [1958], an increasing number of explicitly formulated hypotheses have been tested using these data. The distinguishing feature of the so-called New Economic History is just this juxtaposition of explicitly formulated hypotheses and historical data.

This book is an introduction to the new literature as it pertains to American economic growth in the nineteenth century. The new data will be surveyed and several of the hypotheses that have been advanced to explain them will be examined. In addition, the impact on economic growth of several institutions prominent on the American scene will be discussed.

Because the use of explicit hypotheses and models is the most striking aspect of this work, the present study concentrates on it. (Readers interested in the sources of the data are referred to the Cited Sources.) One cannot present the conclusions that emerge from these models without a taste of the reasoning behind them, since the conclusions cannot be evaluated without an understanding of the methodology used to derive them. A few articles therefore have been selected for extensive discussion, and the limitations of space have been interpreted as limitations on the number of such descriptions as opposed to the length of each. The articles chosen are both important and amenable to analytic summary. The discussion here will be highly critical in order to show the reader the kinds of questions that should be raised about even the most tightly reasoned piece, but the criticisms should not be allowed to obscure the enormous contribution to our historical understanding made by these important articles. 